FINAL TECHNICAL REPORT

\title{
Dictyostelium discoideum Cellulose Synthase: Structure/Function Analysis and Identification of Interacting Proteins
}

\author{
Richard L. Blanton, PI/PD \\ Period of Report: \\ 15 August 1999 to 14 August 2003 \\ Recipient Organization: Texas Tech University \\ Lubbock, Texas 79409 \\ Note that Dr. Blanton's current address is: \\ Department of Botany \\ North Carolina State University \\ Campus Box 7612 \\ Raleigh, NC 27695-7612 \\ (919) 513-4074 \\ Larry_Blanton@ncsu.edu
}

Award Number: $\quad$ DE-FG-03-99ER30335

Residual Funds: $\quad \$ 1,684.86$

\section{Publications Attributable to DOE Funding}

Blanton, R.L., D. Fuller, N. Iranfar, M.J. Grimson, and W.F. Loomis. 2000. The cellulose synthase gene of Dictyostelium. Proceedings of the National Academy of Sciences (U.S.A.) 97: 2391-2396.

Zhang, P., A.C. McGlynn, W.F. Loomis, R.L. Blanton, and C.M. West. 2001. Spore coat formation and timely sporulation depend on cellulose in Dictyostelium. Differentiation 67: 72-79.

Levraud, J.P.., M. Adam,, M.F. Luciani, C. de Chastellier, R.L. Blanton, and P. Golstein. 2003. Dictyostelium cell death: early emergence and demise of highly polarized paddle cells. Journal of Cell Biology 160: 1105-1114.

\section{Abstracts Reporting Work Attributable to DOE Funding}

Grimson, M.J. and R.L. Blanton. 2000. Patterns of cellulose deposition in the developing stalk of Dictyostelium discoideum. International Dictyostelium Conference, Dundee, Scotland.

[DOE-funded work was presented at the 2000 Gordon Conference on plant cell walls, in Merident, NH, where I was an invited speaker. However, no abstracts are published for Gordon conferences.] 


\section{Summary}

The major accomplishments of this project were: (1) the initial characterization of $d c s A$, the gene for the putative catalytic subunit of cellulose synthase in the cellular slime mold Dictyostelium discoideum (Blanton et al., 2000); (2) the detection of a developmentally regulated event (unidentified, but perhaps a protein modification or association with a protein partner) that is required for cellulose synthase activity (i.e., the $d c s A$ product is necessary, but not sufficient for cellulose synthesis) (Blanton et al., 2000); (3) the continued exploration of the developmental context of cellulose synthesis and DcsA (Zhang et al., 2001; Levraud et al., 2003); (4) the isolation of a GFP-DcsA-expressing strain (work in progress); and (5) the identification of Dictyostelium homologues for plant genes whose products play roles in cellulose biosynthesis (work in progress). Although our progress was slow and many of our results negative, we did develop a number of promising avenues of investigation that can serve as the foundation for future projects.

\section{Background}

The initial characterization of $d \operatorname{cs} A$ was performed in collaboration with Dr. William F. Loomis (University of California-San Diego; Blanton et al., 2000). This work began prior to the start of the project period (indeed, our preliminary results figured prominently in the proposal), but continued well into the first year of the project, Dr. Loomis's lab isolated $\operatorname{dcs} A$ as part of a large NIH-funded project (the Dictyostelium Developmental Gene Program; http://glamdring.ucsd.edu/others/dsmith/dictydb.html) using restriction-enzyme mediated insertional mutagenesis (REMI; Kuspa and Loomis, 1992) to identify important developmentally regulated genes (Loomis et al., 1994). DcsA was identified very late in that project, as indicated by the strain numbers of the insertional mutant: DG1099 (the $99^{\text {th }}$ gene found in the project). A second, independent insertional mutant was subsequently identified, DG1128, and the insertion determined to be in $d c s A$. Additional strains we had to work with were TL127, a strain in which dcs A was ablated by homologous recombination, and TL128, a strain in which cellulose synthesis was "rescued" by transformation with the cloned gene under the control of a standard "constitutive" promoter (Cohen et al., 1986). Additional strains of experimental interest included HMX44dcs $A$. HMX44 is a derivative of the strain used in my lab to study developmental regulation of cellulose synthesis in stalk cell monolayers (Blanton, 1993). It is noteworthy because amoebae plated as monolayers will differentiate into stalk cells (and hence initiate cellulose synthesis) upon addition of the stalk-cell inducing chemical DIF-1 (HM44 cannot synthesize DIF-1, but is responsive to it). Hence, HM44 monolayer cultures represent an inducible cellulose-synthesizing experimental system. To summarize:

Table of Strains

\begin{tabular}{|l|l|l|}
\hline \multicolumn{1}{|c|}{ Strain } & \multicolumn{1}{|c|}{ Parent } & \multicolumn{1}{c|}{ Characteristics } \\
\hline DG1099 & $\begin{array}{l}\text { AX4 (axenic mutant derived from } \\
\text { wild-type strain) }\end{array}$ & $d c s A$ null by insertional mutagenesis \\
\hline DG1128 & AX4 & $\begin{array}{l}d c s A \text { null by insertional } \\
\text { muatagenesis }\end{array}$ \\
\hline TL127 & AX4 & $\begin{array}{l}d c s A \text { null by homologous } \\
\text { recombination }\end{array}$ \\
\hline TL128 & DG1128 & $\begin{array}{l}\text { "rescue" strain; cloned } d c s A \text { under } \\
\text { the control of a } \\
\text { "constitutive"promoter }\end{array}$ \\
\hline
\end{tabular}


Table of Strains, cont'd

\begin{tabular}{|l|l|l|}
\hline \multicolumn{1}{|c|}{ Strain } & \multicolumn{1}{|c|}{ Parent } & \multicolumn{1}{c|}{ Characteristics } \\
\hline HM44 & V12M2 ("wild type") & $\begin{array}{l}\text { Chemically mutagenized strain that } \\
\text { is deficient in production of DIF-1, } \\
\text { but responds to exogenously added } \\
\text { DIF-1 }\end{array}$ \\
\hline HMX44 & Axenic mutant of HM44 \\
\hline HMX44dcsA & HMX4 & $\begin{array}{l}\text { dcsA null strain by homologous } \\
\text { recombination }\end{array}$ \\
\hline
\end{tabular}

TL128 provided valuable confirmation of the role of DcsA in cellulose synthesis. In addition, our detailed analysis of this strain yielded an exciting result. The Dictyostelium promoter used in the expression vector for the rescue transformation is the commonly used Actin15 promoter, which is often referred to as constitutive. In fact, the expression pattern of Actin 15 is constitutive from amoebae through aggregation, but then tapers off significantly during later development (Cohen et al., 1986). In contrast, $d c s A$ is not expressed in amoebae and reaches a peak during mid- to late development (Blanton et al., 2000). RNA blots of extracts from TL128 showed a constant level of $d c s A$ mRNA throughout all stages of development (including amoebae; Blanton et al., 2000). Immunoblots using an antibody to a peptide derived from the DcsA sequence showed constant levels of DcsA protein throughout development (including amoebae; Blanton et al., 2000). However, the pattern of cellulose synthase activity was essentially the same as that of the wild type (i.e., no cellulose synthase activity in amoebae and aggregating cells; Blanton et al., 2000). Therefore, the presence of DcsA alone was not sufficient for cellulose synthesis to occur. Instead, a developmentally regulated event (a modification of DcsA, a targeting event, or the appearance of other proteins) was required for cellulose synthesis to occur. The TL128 "rescue" strain was developed after the submission of the proposal, but this result directly impacted the project by suggesting a number of experiments and impacting the design of our proposed heterologous expression experiments.

It is important to remember throughout the discussion that follows that a great strength of Dictyostelium for cellulose synthase studies is the existence of a reliable, high-rate, in vitro assay for cellulose synthase activity (Blanton and Northcote, 1990). This means that we were not restricted to in vivo analyses of mutant strains; in vitro studies using membrane preparations were also possible.

\section{Status of Individual Goals Stated in Original Project Abstract (presented in an order different from that in original abstract)}

\section{Test for functionality of plant and bacterial catalytic subunits in Dictyostelium}

The exciting advances in the study of plant cellulose synthases suggested that a major contribution could be made by developing Dictyostelium as a heterologous expression host for plant cellulose synthases, not only to generate sufficient quantities of proteins for crystallographic and biochemical studies, but also to help identify interacting proteins and enable studies of protein targeting and rosette assembly. Dictyostelium amoebae do not express $d c s A$ and so are an appropriate host for these kinds of studies. There was precedent for their use as expression hosts for heterologous membrane proteins (Cohen et al., 1996; Chaumont et al., 
1997), their glycosylation mechanisms are much more similar to plants than is that of yeast (Slade et al., 1997), and my laboratory had extensive experience in freeze-fracture studies of Dictyostelium (Grimson et al., 1996). We were also interested to see if cellulose synthesis could be "rescued" in the $d c s A$ null strains by the heterologous cellulose synthases.

At the time there were no generally available antibodies to the cellulose synthase. This presented the challenge of identifying the presence of the expressed protein. Our initial Dictyostelium expression vectors had myc tags, which would enable detection of expressed proteins by antibody-based detection systems.

We obtained full length cDNA clones of the catalytic subunits of cellulose synthase from cotton (Gossypium hirsutum), Acetobacter xylinum, and Agrobacterium tumefasciens. These were cloned into an actin15-driven expression vector and transformed into Dictyostelium cells using electroporation. Our initial efforts had very low transformation rates, so we spent considerable time modifying conditions, trying other transformation methods, recloning $d c s A$ into different expression vectors, discussing the problem with a number of laboratories, and even purchasing a different electroporator, when suspicions were cast on the brand of instrument we were using initially. We eventually managed to obtain reasonable numbers of colonies of transformants, but none of those showed signs of expression of the cellulose synthases. Our alarm at this situation increased when we repeated the rescue transformation with the $d \operatorname{cs} A$ expression plasmid. We were able to isolate rescued strains, but at very low frequencies amongst the transformant colonies.

We were facing two problems: (1) low transformation frequency; and (2) low expression frequency among the transformants. Neither of these could have been anticipated. My laboratory had long experience with Dictyostelium transformations (albeit not with protein expression vectors, but rather with disruption vectors and reporter gene fusion vectors) and had never had problems with transformation frequency. Other laboratories had reported success with expression of large membrane proteins (both from Dictyostelium and other organisms). After tackling this frustrating problem for over a year, we decided to move on to other more promising avenues.

At the very end of the project, I discovered two problems that contributed to our difficulties: (1) The postdoctoral fellow doing these experiments had chosen to follow protocols leading to vector integration. In fact, for the kinds of studies we were doing, it would have been better to use the procedures whereby the vector remained independent, which leads to higher copy numbers and hence greater levels of protein expression than can be obtained with nonintegrating vectors. We had tried non-integrating conditions early on without success - but that was likely due to our transformation frequency travails; and (2) The strains we were using were all in an Ax4 background. Although used to great effect by Dr. Loomis's laboratory, Ax4 is found by many labs to be problematic in transformation experiments. As discussed later, I obtained $d s c A$ null strains made in other backgrounds and had much greater success with transforming them. Of the two problems, this second one proved to be the most serious. Even when we used our best transformation conditions with non-integrating vector conditions our results with AX4-derived strains were still poor.

2. Perform a detailed structure/function analysis of the cellulose synthase using a random mutagenesis strategy to identify regions of the cellulose synthase critical for function in the cell and site-directed mutagenesis to confirm the roles of certain conserved amino acids. 
We had hoped to create a variety of strains altered in various aspects of cellulose synthesis in an effort to unravel the mechanism of cellulose biosynthesis and to find clues concerning the proteins with which the cellulose synthase interacts. In such an experiment, a $d c s A$ null strain would be transformed by a library of altered synthases (created by PCR-mutagenesis). Transformants would be dilution plated on bacterial lawns to allow single cells to multiply clonally. If sufficiently diluted, these colonies remain separated until the cells in the middle develop. Visual examination or the use of cellulose-detecting fluorochromes in the medium would allow the rapid identification of cells that are synthesizing cellulose (positive for the fluorochrome) but in some kind of altered way (as detected by developmental irregularities). In vitro assays of membrane preparations would be another means to identify altered activity, although difficult to perform on a large scale. Our difficulties with transformation frequency, and more importantly, protein expression, rendered these experiments unfeasible. The few times we tried this all the transformant colonies were negative for cellulose. We were beginning to lose confidence that any protein was being expressed in the transformants (as discussed above), so did not try the heroic mass-scale in vitro assays.

Other developments discouraged our pursuing this line of investigation. At its best, this approach would have given us clues for what to pursue in a subsequent project. This kind of random approach made sense when originally proposed. We were still at the stage of knowing almost nothing about the proteins involved in cellulose synthesis in plants. However, rapid progress began to be made and a number of potential partners for the cellulose synthase in plants identified. It seemed more appropriate to use the information from plants to find homologues in Dictyostelium as its full genome sequence was produced. In that way, Dictyostelium could be helpful in confirming and defining a role for those proteins in cellulose synthesis. This approach is discussed further below.

3. Search for interacting proteins using protein affinity chromatography, coimmunopreciptiation, and yeast two-hybrid methods.

A key tool here was expected to be the antibody produced to the $\mathrm{U} 2$ region of the cytoplasmic loop of DcsA, which had been used to generate the developmental time course immunoblot in Blanton et al. (2000). Alas, the anti-U2 antibody did not work in light- or electron-microscope immunocytochemistry. Repeated immunoprecipitation experiments were performed, but never yielded anything of interest.

We decided to generate another antibody, this time to the entire cytoplasmic loop, hoping that would encompass additional and more accessible antigenic sites. The effort to generate and purify the resulting fusion protein extended across two postdocs and involved significant technical difficulties, including cryptic initiation codons, non-expression, and insoluble inclusion bodies. We were close to success when the second postdoc left to assume an assistant professorship. The bacterial strains with the appropriate vector are in storage and I plan to return to their analysis and the generation of the fusion protein in the near future.

Yeast two-hybrid has been performed with success in Dictyostelium (e.g., Skruzny et al., 2001). However, given the fact that $\operatorname{dcs} A$ is a membrane protein and the kind of effort that would have to be expended to make this work, it seemed some of the initiatives described below would be more fruitful. It also seemed that the yeast two-hybrid would be more useful when dealing with specific suspected partners rather than as a random method. 


\section{Additional Goals Identified and Pursued during the Course of the Project}

\section{Attempting to activate the ectopically expressed $d c s A$}

We were intrigued by the result with the rescue strain, TL128: $d c s A$ was expressed at high levels in amoebae and aggregating cells (wild type cells do not express it at all in those stages) and yet cellulose synthase activity as measured by an in vitro assay followed the wild type pattern. One explanation could be that a developmentally regulated protein modification was required to activate the synthase. We tested for this by a variety of mixing experiments. Membrane preparations or whole cell lysates were prepared from TL128 amoebae. These were mixed with whole cell lysates or supernatants from late developmental stage null strains (which would presumably have the modifying activity, even if lacking the synthase). Variations to the procedure included mix ratios, preincubation times, and pretreatment with proteases, phosphatases, kinases, protease inhibitors, phosphatase inhibitors, and kinase inhibitiors. Membrane-mixing experiments were also performed. In no case could we obtain activity from the DcsA-containing TL128 membranes.

\section{Continuing to explore the developmental regulation of cellulose biogenesis}

My lab has a long history of exploring the developmental regulation of cellulose biogenesis in Dictyostelium (Blanton, 1993; Grimson et al., 1996; Blanton, 1997). An intriguing observation from our initial studies of development in the $d c s A$ null strains was that the developing structures displayed all of the normal cell movements associated with late development; i.e., the cells did not detect the absence of cellulose and development was not arrested (Blanton et al., 2000).

In collaboration with the laboratory of Dr. C.R. West (then of the University of Florida), we found that in spore development the absence of cellulose is detected by the pre-spore cells, which are delayed in their development in the $\operatorname{css} A$ null strain. Their eventual development is not normal, because of the absence of cellulose. The delay in development could be overcome by addition of 8-Br-cAMP to activate intracellular protein kinase A (PKA), an event normally induced by a factor released by prestalk cells during late development. The absence of cellulose produced by the prestalk cells may result in the non-production or inappropriate localization of the PKA-inducing signal. Once induced, the prespore cells secrete the protein components of their spore coats, which depend upon cellulose for their proper localization into a stratified cell wall (Zhang et al., 2001).

In collaboration with the laboratory of Dr. P. Golstein (Centre d'Immunologie de Marseille-Luminy, C.N.R.S., Marseille, France), we examined the effect of the absence of cellulose on stalk cell development in monolayers. In $d c s A^{+}$cells, the stalk cells in monolayers follow a clearly defined sequence of cell shape changes and internal modifications leading to the formation of a cellulose-encased, vacuolated, and non-viable stalk cell. In HMX44dcs $A^{-}$strain the absence of cellulose has no effect upon this sequence of events. In the end, the cells simply lyse, releasing their contents into the medium (Levraud et al., 2003).

\section{Development of DcsA-GFP strains}

Monolayer cultures provide the opportunity to study cellulose synthesis by a single cell (Blanton, 1993). A GFP-tagged version of DcsA would thereby allow direct microscopical 
observation of the movement of cellulose synthases during cellulose synthesis. We attempted to generate both N-terminal and C-terminal versions, succeeding first with the former. DcsA does not have an N-terminal cleaved signal sequence, so there was no a priori reason why this would not work. At this time we faced two challenges: (1) we had not overcome our transformation and expression problems; and (2) the best monolayer-performing strains are not transformable. However, the existence of the GFP-tagged DcsA had motivated the PI/PD to return again to the problem of transformation and expression. He managed to obtain high transformation/high expression of the N-terminal GTP-tagged DcsA into a $d \operatorname{cs} A$-null strain developed by insertional mutagenesis in a strain that could be persuaded to form stalk cells in monolayers (although not at high levels). We had just reached the point of beginning our confocal microscope studies of this strain when the PI/PD relocated to NC State and the grant terminated. We have not completed all of the necessary experiments confirming the expression of the GFP-DcsA (i.e., RNA blots, immunoblots). We also need to confirm that the tag remains on the functional protein.

\section{The identification of genes whose products might play roles in cellulose biosynthesis}

Genetic studies in Arabidopsis have identified a number of proteins that have some role in cellulose synthesis. The Dictyostelium genome has now been completely sequenced. Not only does this confirm that there is a single gene for the cellulose synthase catalytic subunit in this organism $(\operatorname{dcs} A)$, but it also provides the opportunity to identify potential homologues in Dictyostelium to the proteins identified in plants. The genes for these can then be cloned, used to construct knock-out vectors, and their necessity for cellulose synthesis confirmed. We had just begun this process in the last year of the grant. We successfully cloned the Dictyostelium sterol glucosyltransferase (identified as having a role in cellulose synthesis in plants by Peng et al. (2002) and performed quantitiative RT-PCR to confirm that its expression pattern was consistent with its being involved in cellulose synthesis.

To facilitate the gene disruption, we began to work with a new homologous recombination technique that promises to lead to more efficient generation of knock-out plasmids (Abe $e t$ al., 2003). Other candidates with plant homologues were identified, including endoglucanases (identified as having a role in cellulose synthesis in plants by Lane et al., 2001)) and chitinases (identified as having a role in cellulose synthesis in plants by Zhong et al., 2002). We designed primers for all of the Dictyostelium endoglucanase and chitinase homologues and were in the midst of the RT-PCR experiments (without early success-in contrast to the sterol glucosyltransferase, which worked the first time) when the grant came to an end and the lab closed with the PI/PD's move to NC State. Clearly this is a promising approach and one that we will continue.

\section{Conclusion}

This project faced a number of significant technical hurdles, pursued several important questions that ended with negative results, and suffered from a number of personnel problems that adversely impacted our ability to attain the goals stated in the original abstract. However, we did manage to make some significant contributions and the groundwork has been laid for future success: we have GFP-tagged strains to exploit and potential protein partners to study. Dictyostelium continues to enjoy a number of advantages for the study of cellulose synthesis: there is a single gene for the cellulose synthase subunit, a reliable, high-rate in vitro assay system, an inducible, single-cell monolayer culture system, and a large body of knowledge 
concerning basic cellular and developmental mechanisms. A key goal for the future is to link some aspect of the cellulose synthesizing machinery into those well-known mechanisms, which would allow rapid progress towards understanding the cellular and developmental context of cellulose synthesis.

\section{References}

Abe, T., J. Langenick, and J.G. Williams. (2003). Rapid generation of gene disruption constructs by in vitro transposition and identification of a Dictyostelium protein kinase that regulates its rate of growth and development. Nucl. Acids Res. 31: E107 (10 pages).

Blanton, R. L. (1993). Prestalk cells in monolayer cultures exhibit two distinct modes of cellulose synthesis during stalk cell differentiation in Dictyostelium. Development 119: 703-710.

Blanton, R. L. (1997). Cellulose biogenesis in Dictyostelium discoideum. In Dictyostelium - A model system for cell and developmental biology. Y. Maeda, K. Inouye, and I. Takeuchi, eds. Tokyo, Japan, Universal Academy Press: 379-391.

Blanton, R. L., D. Fuller, N. Iranfar, M.J. Grimson, and W.F. Loomis. (2000). The cellulose synthase gene of Dictyostelium. Proc. Natl. Acad. Sci. USA 97: 2391-2396.

Blanton, R. L. and D. H. Northcote (1990). A 1,4-ß-D-glucan synthase system from Dictyostelium discoideum. Planta 180: 324-332.

Chaumont, F., W. F. Loomis, and M.J. Chrispeels. (1997). Expression of an Arabidopsis plasma membrane aquaporin in Dictyostelium results in hypoosmotic sensitivity and developmental abnormalities. Proc. Natl. Acad. Sci. USA 94: 6202-6209.

Cohen, N. R., D. A. Knecht, and H.F. Lodish. (1996). Functional expression of rat GLUT 1 glucose transporter in Dictyostelium discoideum. Biochem. J. 315: 971-975.

Cohen, S. M., D. Knecht, H.F. Lodish, and W.F. Loomis. (1986). DNA sequences required for expression of a Dictyostelium actin gene. EMBO J. 5: 3361-3366.

Grimson, M. J., C. H. Haigler, and R.L. Blanton. (1996). Cellulose microfibrils, cell motility, and plasma membrane protein organization change in parallel during culmination in Dictyostelium discoideum. J. Cell Sci. 109: 3079-3087.

Kuspa, A. and W. F. Loomis (1992). Tagging developmental genes in Dictyostelium by restriction enzyme-mediated integration of plasmid DNA. Proc. Natl. Acad. Sci. USA 89: 8803-8807.

Lane, D. R., A. Wiedemeier, L. Peng, H. Hofte, S. Venhettes, T. Desprez, C.H. Hocart, R.J. Birch, T.I. Baskin, J.E. Burn, T. Arioli, A.S. Betzner, and R.E. Williamson. (2001). Temperature-sensitive alleles of RSW2 link the KORRIGAN endo-1,4- 3 -glucanase to cellulose synthesis and cytokinesis in Arabidopsis. Plant Physiology 126(1): 278-288.

Levraud, J. P., M. Adam, M.F. Luciani, C. de Chastellier, R.L. Blanton, and P. Golstein. (2003). Dictyostelium cell death: early emergence and demise of highly polarized paddle cells. $J$. Cell Biol. 160: 1105-1114.

Loomis, W. F., A. Kuspa, and G. Shaulsky. (1994). Gene discovery in Dictyostelium. Genetic Engin. 16: 49-64.

Peng, L., Y. Kawagoe, P. Hogan, and D. Delmer. (2002). Sitosterol-ß-glucoside as primer for cellulose synthesis in plants. Science 295: 147-150.

Skruzny, M., M. Ambrozkova, I. Fukova, K. Martinkova, A. Blahuskova, L. Hamplova, F. Puta, and P. Folk. (2001). Cyclophilins of a novel subfamily interact with SNW/SKIP 
coregulator in Dictyostelium discoideum and Schizosaccharomyces pombe. Biochim. Biophys. Acta 1521: 146-151.

Slade, M. B., K. R. Emslie, and K.L. Williams. (1997). Expression of recombinant glycoproteins in the simple eukaryote Dictyostelium discoideum. Biotechnol. Genetic Engin. Rev._14: 135.

Zhang, P., A.C. McGlynn, W.F. Loomis, R.L Blanton, and C.M. West. (2001). Spore coat formation and timely sporulation depend on cellulose in Dictyostelium. Differentiation 67: 72-79.

Zhong, R., S.J. Kayes, B.P. Shroeder, and Z.H. Ye. (2002) Mutation of a chitinase-like gene causes ectopic deposition of lignin, aberrant cell shapes, and an overproduction of ethylene. Plant Cell 14: 165-179. 